\title{
Three new species of the leafhopper genus Dayus Mahmood from China (Hemiptera, Cicadellidae,Typhlocybinae, Empoascini)
}

\author{
Xiaofei Yu ${ }^{1,2, \dagger}$, Maofa Yang ${ }^{1,2, \ddagger}$ \\ I Institute of Entomology, Guizhou University, Guiyang Guizhou, 550025, P. R. China 2 Guizhou Provincial \\ Key Laboratory for Agricultural Pest Management of the Mountainous Region, Guiyang Guizhou, 550025, \\ P. R. China \\ † http://zoobank.org/872D7371-CDF4-4044-95DF-B2705F6293E2 \\ ‡ http://zoobank.org/79B3BA54-5CBD-43CD-B50C-FAABBBFA4904 \\ Corresponding author: Maofa Yang (yangmaofa@sohu.com)
}

Academic editor: MickWebb | Received 19September 2013 | Accepted 18 November 2013 | Published 25 November 2013

http://zoobank.org/9412C02F-6433-4090-BF17-18A280B90782

Citation: Yu XF, Yang MF (2013) Three new species of the leafhopper genus Dayus Mahmood from China (Hemiptera, Cicadellidae, Typhlocybinae, Empoascini). ZooKeys 355: 1-8. doi: 10.3897/zookeys.355.6277

\section{Abstract}

Three new species of the Oriental empoascine leafhopper genus Dayus Mahmood are described from China: D. bifurcatus sp. n., D. trifurcatus sp. n. and D. serratus sp. n. A key to distinguish all Chinese species of the genus is provided.

\section{Keywords}

Auchenorrhyncha, leafhopper, taxonomy, morphology

\section{Introduction}

The Oriental typhlocybine leafhopper genus Dayus was established by Mahmood in 1967 with D. elongatus Mahmood (Singapore) as its type species. Subsequently, Dworakowska (1971) described D. takagii Dworakowska (Japan) and transferred D. upoluanus (Osborn, 1934) (Western Samoa) and D. euryphaessus (Kirkaldy, 1907) (Fiji) to the geuns, Dworakowska and Viraktamath (1978) added a new species: D. formosus from China (Taiwan) and Qin and Zhang (2007) added three new species from China: D. lii Qin \& Zhang, D. membranaceus Qin \& Zhang and D. lamellatus Qin \& Zhang. 
Here we describe three new species of Dayus from China and provide a key for the separation of all known Chinese species. The specimens examined are deposited in the Institute of Entomology, Guizhou University, Guiyang, Guizhou, China (GUGC) and The Natural History Museum, London (BMNH).

\section{Materials and methods}

The methods and terminology follow Zhang (1990) except for the nomenclature of wing, for which we follow Dworakowska (1993). Male specimens were dissected under a MOTIC B1 SMS-168 SERIES microscope. Figures were made using an OLYMPUS CX41 and enhanced using Adobe Illustrator CS4. Pictures were taken with VHX$1000 \mathrm{C}$ and dealt with by Adobe Illustrator CS4. The body length is measured from the apex of the head to the apex of the forewing.

\section{Results}

\section{Genus Dayus Mahmood}

http://species-id.net/wiki/Dayus

Dayus Mahmood, 1967: 39.

Type species. Dayus elongatus Mahmood, 1967 by original designation.

Diagnosis. Vertex (Fig. 1) slightly longer medially than next to eye. Forewing (Fig. 22) with $3^{\text {rd }}$ apical cell petiolate, cua cell broad distally; veins RP, MP' and MP" $+C u A$ ' arise from $\mathrm{m}$ cell. Hindwing (Fig. 23) with apically broad $\mathrm{m}$ cell. Male pygofer (Figs 3, $13,24)$ abruptly and strongly narrowing caudad; dorsal bridge about half length of pygofer (Figs 15, 26); with few rigid microsetae distally; elongate ventral appendage present, extended beyond pygofer. Subgenital plate (Figs 5, 17, 27) with basal group of macrosetae and one or two oblique rows of more distal macrosetae. Connective (Figs 7, 19, 29) completely fused with the base of aedeagus. Aedeagus (Figs 8, 20,30) with basal apodeme absent; shaft strongly curved posteriorly at base with one or two pairs of processes.

\section{Key to the Chinese species (males)}

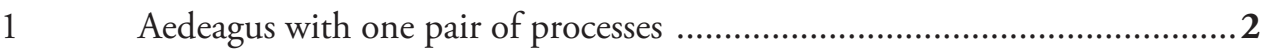

- $\quad$ Aedeagus with two pairs of processes …….................................................... 6

2 Aedeagal processes with basal serrated lobes (Figs 29-30) ...D. serratus sp. n.

- $\quad$ Aedeagal processes not dentate basally ....................................................... 3

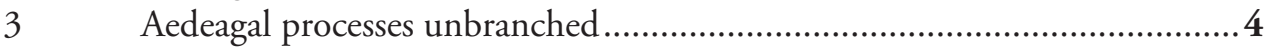

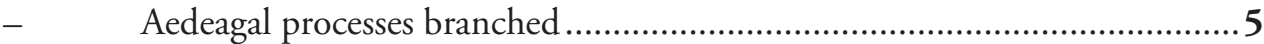


4 Aedeagal processes arising near midlength of shaft D. membranaceus

- $\quad$ Aedeagal processes arising at apex of shaft

D. formosus Aedeagal processes trifurcate, subapical on shaft (Figs 19-20)

D. trifurcatus sp. $\mathbf{n}$.

- $\quad$ Aedeagal processes bifurcate, at apex of shaft (Figs 7-8) ....D. bifurcatus sp. n. 6 Aedeagal processes bifurcate; apical pygofer ventral appendage branched ....D. lii - Aedeagal processes not bifurcate; apical pygofer ventral appendage unbranched .7

7 Apical aedeagal processes straight, subapical processes leaf-like.....D. lamellatus - $\quad$ Apical aedeagal processes hook-shaped, subapical processes slim.... D. takagii

\section{Dayus bifurcatus Yu \& Yang, sp. n.}

http://zoobank.org/8636DDEB-EDC7-4A8C-B6EA-6D7AEF8756DC http://species-id.net/wiki/Dayus_bifurcatus

Figures 1-9

Description. Length, male $3.0 \mathrm{~mm}$.

General color reddish to reddish brown. Both sides of coronal suture with a brownish patch.

Male ventral abdominal apodemes reaching segment 5 (Fig. 4). Male pygofer with dorsoposterior margin sinuate; pygofer appendage with dorsal margin sinuate in lateral view, tapering to apex (Fig. 3). Subgenital plate about twice as broad basally than distally, with three lateral macrosetae in basal group, an oblique line of 14 macrosetae and several long fine setae subbasally to apex and ca.12 short microsetae at outer margin (Fig. 5). Paramere as in Fig. 6. Aedeagus shaft long and narrow, slightly depressed dorsoventrally, similar in width throughout length in ventral view, with a pair of short bifurcate apical processes (Figs 7, 8). Anal tube process short (Fig. 9).

Type material. Holotype male. China: Zhejiang Province, Fengyang mountain, 30 July 2009, coll. Junqiang Ni.

Etymology. The new species name alludes to the pair of apical bifurcate aedeagal processes.

Remarks. The new species can be distinguished mainly by the shape of the aedeagal shaft and its process configuration as noted in the description.

\section{Dayus trifurcatus Yu \& Yang, sp. n.}

http://zoobank.org/1FCC60EB-60FC-490D-8E7B-7E69594D814C

http://species-id.net/wiki/Dayus_trifurcatus

Figures 10-23

Description. Length, male 4.5-4.6 mm, female $4.7-4.8 \mathrm{~mm}$.

General color yellowish. 
Male ventral abdominal apodemes reaching segment 4 (Fig. 14). Male pygofer with dorsoposterior margin strongly sinuate (Fig. 13); ventral appendage expanded at distal $2 / 3$, thereafter abruptly tapering to spine-like apex (Figs 13,16). Subgential plate abruptly expanded laterobasally about twice as broad basally than distally; with 11 lateral macrosetae in basal group, an oblique line of 17 macrosetae and several long fine setae subbasally to apex and ca.35 short microsetae at outer margin (Fig. 17). Paramere as in Fig. 18. Aedeagus shaft very long and narrow, cylindrical, nearly straight in lateral view, with a subapical trifurcate process on each side, branches slender (Figs 19, 20). Anal tube process relatively long (Fig. 21).

Type materials. Holotype male. China: Beipei, Chongqing, 6 May 2008, coll. Zaihua Yang. Paratypes, $13 \hat{\jmath} \hat{\partial}, 5+90$, same data as holotype (GUGC and $1 \hat{\jmath}, 19$ in BMNH).

Etymology. The new species name alludes to the trifurcate processes of the aedeagus.

Remarks. The new species can be distinguished mainly by the strongly sinuate posterior margin of the pygofer and shape of the aedeagal shaft and configuration of its process as noted in the description.

\section{Dayus serratus Yu \& Yang, sp. n.}

http://zoobank.org/A163BC19-73A4-459A-BA07-125F00EF9D8A

http://species-id.net/wiki/Dayus_serratus

Figures 24-30

Description. Length, male $3.9 \mathrm{~mm}$.

General color yellowish.

Male ventral abdominal apodemes reaching segment 3 (Fig. 25). Male pygofer (Fig. 24) with dorsoposterior margin concave, tapering caudally; ventral appendage with dorsal margin slightly sinuate, tapered to acute apex. Subgenital plate slightly broader basally, with 9 apically rounded lateral macrosetae in basal group, an oblique line of 12 macrosetae and several long fine setae sub-basally to apex and ca.32 short microsetae at outer margin (Fig. 27). Parameres as in Fig. 28. Aedeagus shaft long, basal half strongly dorsoventrally depressed, distal half narrow and cylindrical, serrate laterally at base on dorsal surface; with two long processes arising at midlength on each side of shaft, basally each process with a lateral lamellate serrate lobe (Figs 29, 30). Anal tube process short (Fig. 24).

Type material. Holotype male. China: Hainan Province, Wuzhi mountain, 13 April 2009, coll. Zaihua Yang.

Etymology. The new species name is derived from the serrations at midlength of the aedeagal shaft and base of the aedeagal processes. 
Remarks. The new species can be distinguished mainly by the relatively rather uniform width of the subgenital plate and shape of the aedeagal shaft and configuration of its process as noted in the description.
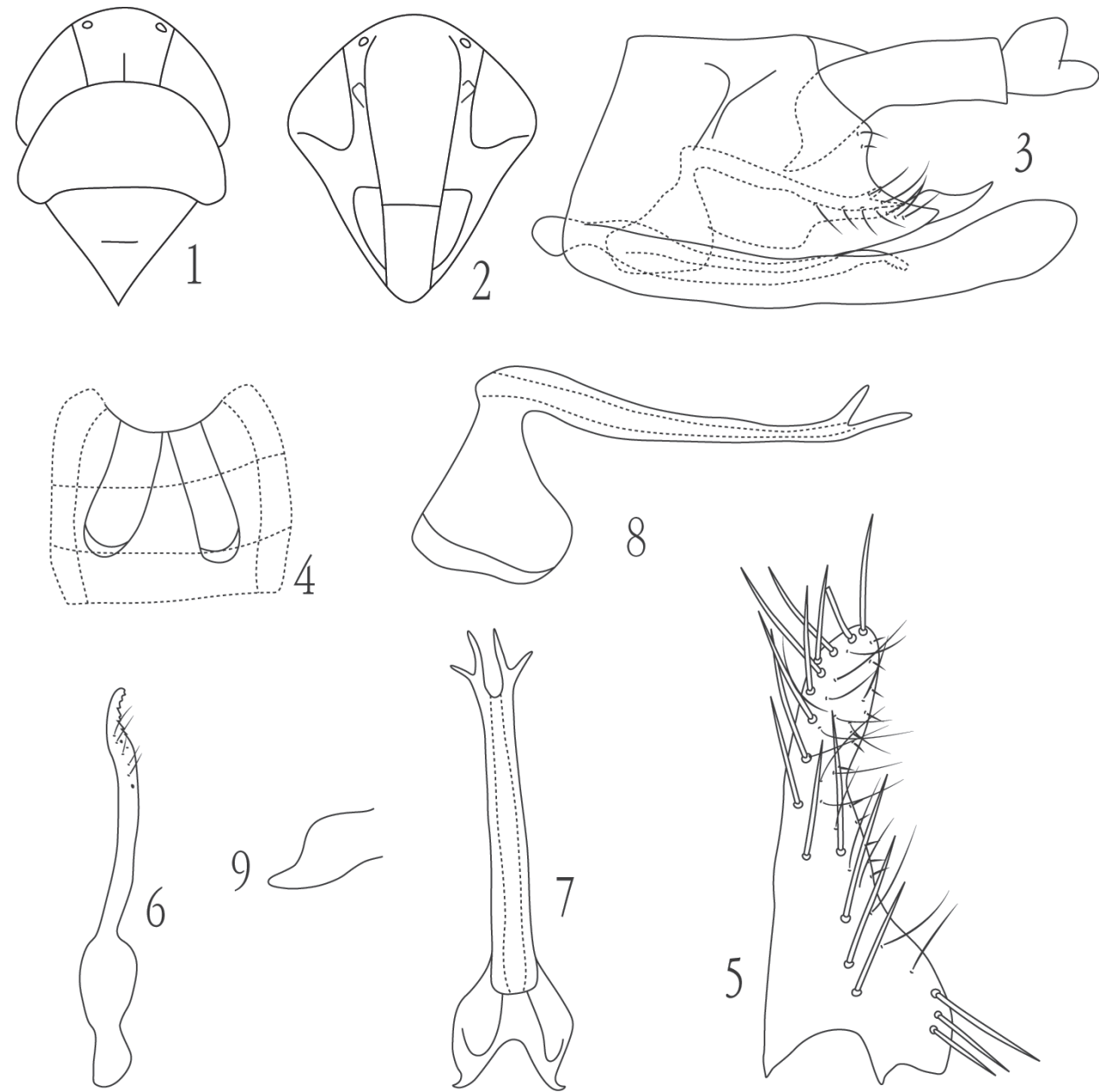

Figures I-9. Dayus bifurcatus Yu \& Yang, sp. n., I head and thorax, dorsal view $\mathbf{2}$ face $\mathbf{3}$ male genital capsule, lateral view $\mathbf{4}$ male abdominal apodemes $\mathbf{5}$ subgenital plate, ventral view $\mathbf{6}$ paramere $\mathbf{7}$ aedeagus and connective, dorsal view $\mathbf{8}$ aedeagus and connective, lateral view $\mathbf{9}$ anal tube process. 

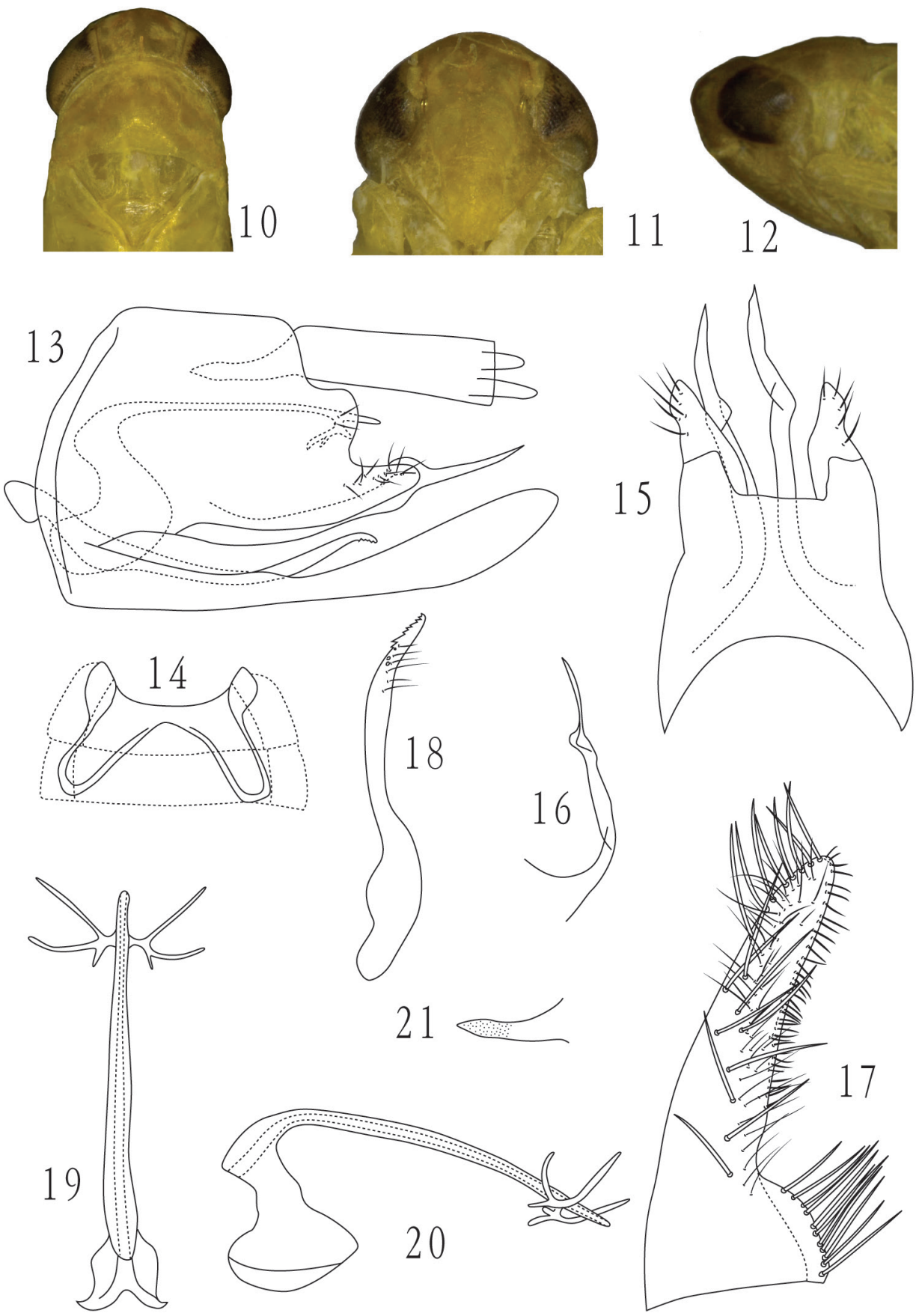

Figures I 0-2 I. Dayus trifurcatus Yu \& Yang, sp. n., I $\mathbf{0}$ head and thorax, dorsal view I I face $\mathbf{I} \mathbf{2}$ head and thorax, lateral view $\mathbf{1 3}$ male genital capsule, lateral view $\mathbf{1} \mathbf{4}$ male abdominal apodemes $\mathbf{1 5}$ male pygofer, dorsal view $\mathbf{1 6}$ ventral pygofer appendage, outside lateral view $\mathbf{1 7}$ subgenital plate, ventral view $\mathbf{1 8}$ paramere $\mathbf{1 9}$ aedeagus and connective, dorsal view $\mathbf{2 0}$ aedeagus and connective, lateral view $\mathbf{2 I}$ anal tube process. 


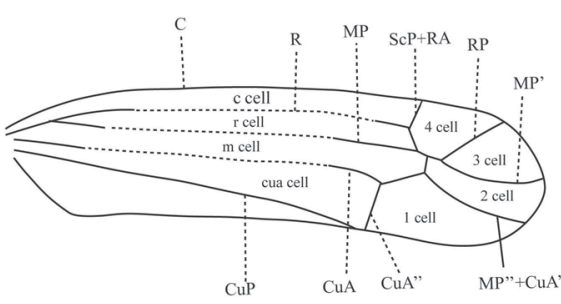

22

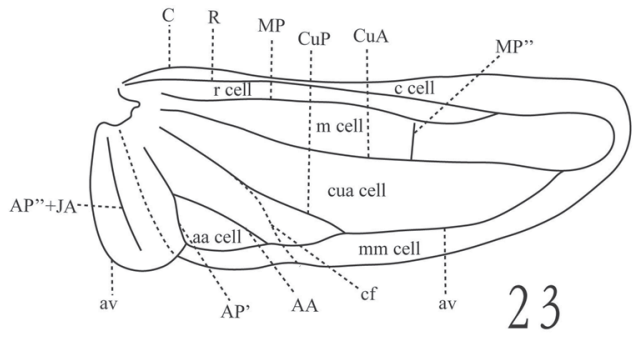

23
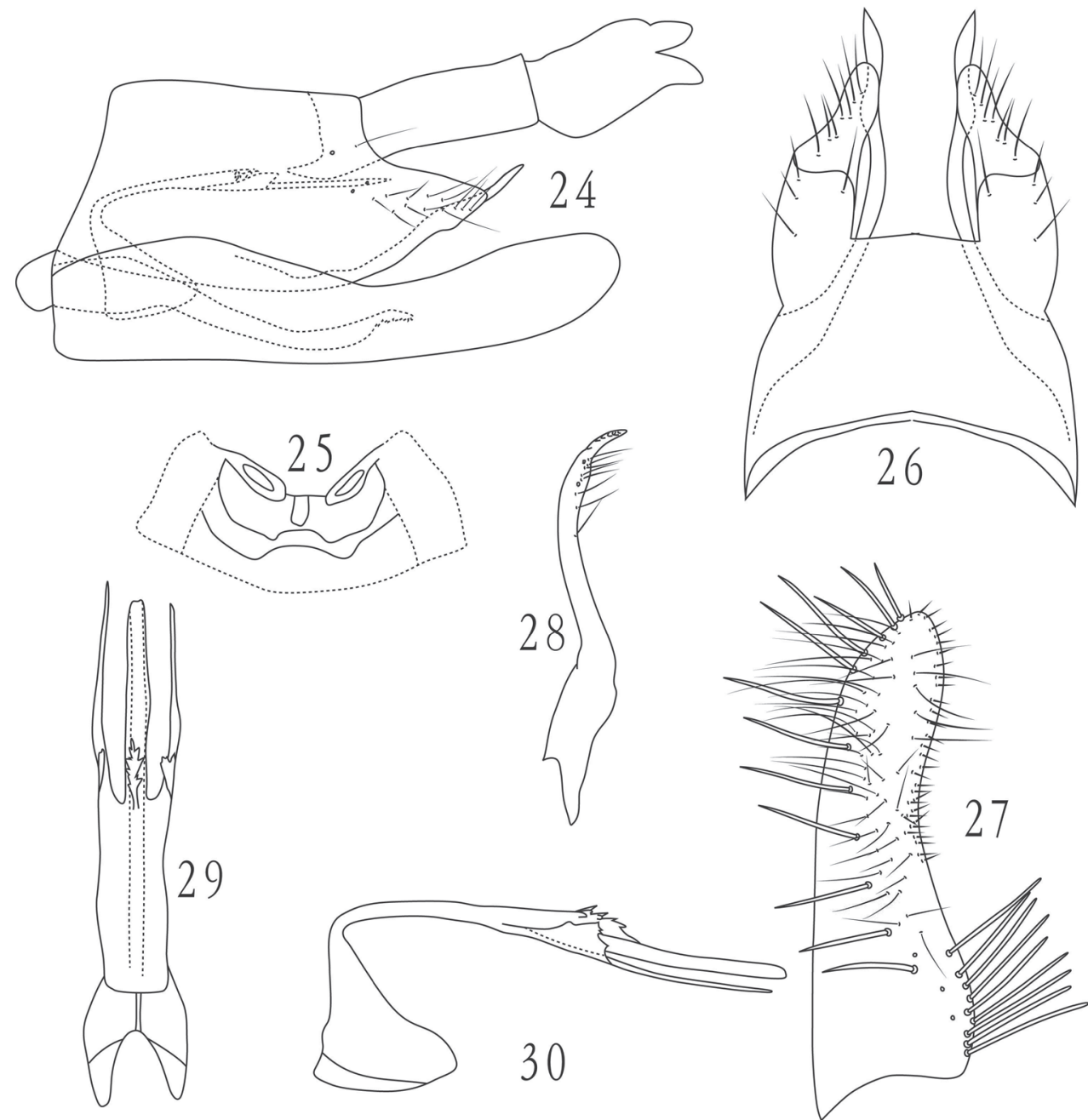

Figures 22-30. 22-23 Dayus trifurcatus Yu \& Yang, sp. n., 22 forewing 23 hind wing 24-30 Dayus serratus Yu \& Yang, sp. n., 24 male genitalia, lateral view $\mathbf{2 5}$ male abdominal apodemes $\mathbf{2 6}$ male pygofer, dorsal view $\mathbf{2 7}$ subgenital plate, ventral view $\mathbf{2 8}$ paramere $\mathbf{2 9}$ aedeagus and connective, dorsal view $\mathbf{3 0}$ aedeagus and connective, lateral view. 


\section{Acknowledgements}

We thank Mr. M. D. Webb (Department of Entomology, The Natural History Museum, England) for reading the manuscript and providing critical comments and kind suggestions. We are very grateful to Dr. Zehong Meng (Guizhou Tea Research Institute, Guiyang, China) and Meng Jiao (Institute of Entomology, Guizhou University, China) for revising the manuscript and taking photos and Prof. Renhuai Dai (Institute of Entomology, Guizhou University, China) for offering valuable literature. Thanks also to Zaihua Yang, Junqiang Ni (Institute of Entomology, Guizhou University, China) for collecting the material used in this study.

\section{References}

Dworakowska I (1971) Dayus takagii sp. n. and some other Empoascini (Auchenorrhyncha: Cicadellidae: Typhlocybinae). Bull. Acad. Pol. Sci. Ser. Sci. Biol. 19(7-8): 501-509.

Dworakowska I, Virktamath CA (1978) On some Indian Typhlocybinae (Auchenorrhyncha: Cicadellidae). Bull. Acad. Pol. Sci. Ser. Sci. Biol. 26(8): 529-548.

Dworakowska I (1993) Remarks on Alebra Fieb. and Eastern Hemisphere Alerini (Auchenorrhyncha: Cicadellidae: Typhlocybinae). Entomotaxonomia 15(2): 91-121.

Mahmood SH (1967) A study of the tyhlocybine genera of the Oriental region (Thailand, the Philippines and adjoining areas). Pacific Insect Monograph 12: 1-52.

Osborn H (1934) Hemiptera. Cicadellidae (Jassidae). Part II. Insects of Samoa and other Samoan terrestrial Arthropoda 4: 163-192.

Kirkaldy GW (1907) Leafhoppers supplement (Hemiptera). Bulletin of the Hawaiian Sugar Planters Association (Entomological Series) 3: 1-186, pl. 1-20.

Qin D, Zhang Y (2007) Revision of the Chinese species of the leafhopper genus Dayus Mahmood (Hemiptera: Cicadellidae: Typhlocybinae: Empoascini), with description of three new species. Zootaxa 1624: 43-51. www.mapress.com/zootaxa/2007f/zt01624p051. pdf

Zhang Y (1990) A taxonomic study of Chinese Cicadellidae (Homoptera). Tianze Press, Shaanxi, 218 pp. 